\title{
Searching for Dark Matter with the Southern Wide-field Gamma-ray Observatory (SWGO)
}

\author{
Aion Viana,, ${ }^{a, *}$ Andrea Albert,,${ }^{c}$ J. Patrick Harding,, ${ }^{c}$ Jim Hinton, ${ }^{b}$ Harm \\ Schoorlemmer, ${ }^{b}$ and Vitor de Souza, ${ }^{a}$ on behalf of the SWGO Collaboration \\ (a complete list of authors can be found at the end of the proceedings) \\ ${ }^{a}$ Instituto de Física de São Carlos, Universidade de São Paulo, Av. Trabalhador São-carlense 400, São \\ Carlos, Brasil. \\ ${ }^{b}$ Max-Planck Institut für Kernphysik, \\ Saupfercheckweg 1, 69117, Heidelberg, Germany \\ ${ }^{c}$ Los Alamos National Laboratory \\ E-mail: aion.viana@ifsc.usp.br
}

Despite mounting evidence that dark matter (DM) exists in the Universe, its fundamental nature remains unknown. We present sensitivity estimates to detect DM particles with a future very-highenergy ( $~ \mathrm{TeV}$ ) wide field-of-view gamma-ray observatory in the Southern Hemisphere, currently in its research and development phase under the name Southern Wide field-of-view Gamma-ray Observatory (SWGO). This observatory would search for gamma rays from the annihilation or decay of DM particles in many key targets in the Southern sky, such as the Galactic halo, several dwarf galaxies, including the promising Reticulum II, and the Large Magellanic Cloud. With a wide field of view and long exposures, such observatory will have unprecedented sensitivity to DM in the mass range of $\sim 100 \mathrm{GeV}$ to a few $\mathrm{PeV}$ from observations of a large fraction of the Galactic halo around the Galactic Center and from Galactic subhalos targets. These results, combined with those from other present and future gamma-ray observatories, will likely probe the thermal relic annihilation cross section of Weakly Interacting Massive Particles for all masses from $\sim 80 \mathrm{TeV}$ down to the $\mathrm{GeV}$ range in most annihilation channels.

$37^{\text {th }}$ International Cosmic Ray Conference (ICRC 2021)

July 12th - 23rd, 2021

Online - Berlin, Germany

\footnotetext{
${ }^{*}$ Presenter
} 


\section{Introduction}

In the past decades a variety of observations solidified our knowledge concerning the presence of dark matter in the universe. These include the temperature anisotropies of the Cosmic Microwave Background Radiations, gravitational lensing, Baryonic Acoustic oscillations, galaxy rotation curves and the compelling fact that dark matter describes the structures and evolution of the universe at all scales (see ref. [1] and references therein). However, these observations rely entirely on the gravitational effects of dark matter and not its fundamental nature. The most attractive candidate to explain DM particles are called Weakly Interacting Massive Particles (WIMPs). These are non-baryonic particles with masses in the $\mathrm{GeV}-\mathrm{TeV}$ range that are thermally produced in the early universe, thus their relic abundance is set by the annihilation cross section. They are stable, electrically neutral, and the right amount of dark matter is produced if their annihilation cross section is at the weak scale.

Dark matter particles populate our universe distributed in dark halos surrounding structures as small as globular clusters to as large as clusters of galaxies. Inside these halos the density may be sufficiently large that pairs of dark matter particles may self-annihilate and produce visible signals of cosmic rays, neutrinos, and gamma rays. The flux originated from dark matter annihilation is proportional to the number density squared, the annihilation cross section, the element of volume in the sky observed, and the energy spectrum. A similar logic applies to decay, which is instead linearly proportional to dark matter density but inversely proportional to the dark matter lifetime [2]. If WIMPs have masses well above the TeV scale, the only near-future discovery possibilities are astrophysical. This is because the current generation of colliders do not reach sufficiently high energies to produce $>\mathrm{TeV} \mathrm{DM}$, and the naturally-produced flux of $\mathrm{DM}$ above the $\mathrm{TeV}$ mass scale is not likely to be reachable with the current target masses and technology of the direct-detection experiments. Several experiments are currently utilised to search for such signatures[3-9] and several more are planned $[10,11]$. Although unambiguous evidence of particle DM is still absent, the allowed parameter space is increasingly constrained by such searches.

Gamma-ray observatories are particularly promising for the DM search at masses beyond 1 $\mathrm{TeV}$, with high sensitivity and sufficient angular resolution obtainable. The main advantages of this powerful indirect detection technique are that gamma rays do not suffer deviation of their trajectories from propagation effects and that characteristic features such as bumps, steps or cut-offs may be present in the energy spectrum, given by the fact that no more energy than DM particle mass per particle can be released in the annihilation of two non-relativistic DM particles. On the other hand gamma rays are also abundantly produced by astrophysical sources in electromagnetic and hadronic processes, thus the unambiguous identification of a DM emission above such astrophysical background is sometimes difficult. However, above the TeV scale, astrophysical gamma-ray backgrounds are expected to be much smaller than at lower energies and allow for detection of very faint DM signals.

Given its large DM content and relative proximity, the Galactic Center (GC) region is expected to be the brightest source of gamma rays from DM annihilation or decay in the sky by several orders of magnitude. Even considering possible signal contamination from other astrophysical sources, it is one of the most promising targets to detect the presence of new massive DM particles. Other attractive targets are dwarf spheroidal galaxies (dSphs). They are some of the most dark-matter- 
dominated objects known, and dozens are known to exist nearby in the Milky Way dark-matter halo. Given their proximity and low astrophysical backgrounds, Milky Way dSphs are excellent targets for searching for gamma-ray emission from dark-matter annihilation or decay.

A TeV gamma-ray observatory with a very wide field of view (FOV) is a promising possibility in the search for extended low-surface brightness emission associated with the DM halos of our own galaxy and its dwarf satellite galaxies. Several observatories of this kind are operational in the Northern Hemisphere (in particular LHAASO [12] and HAWC [3])) using arrays of particle detectors located at high-elevation sites. A similar facility located in the Southern Hemisphere would be highly sensitive to DM gamma-ray signals from the GC region, in particular because the GC would transit close to zenith in relation to such a detector. An observatory of this type has been proposed for construction in South America and recently a collaboration was formed to start the research and development phase under the name Southern Wide field-of-view Gamma-ray Observatory $\left(\mathrm{SWGO}^{1}\right)[13]$. Sensitivity at the highest energies $(>10 \mathrm{TeV})$ is only achievable with a large detection area $\left(>50000 \mathrm{~m}^{2}\right)$ and a long exposure. This can be achieved for the entire observable sky by a passive, wide FOV detector of gamma rays, complementing searches with pointed systems such as Imaging Atmospheric Cherenkov Telescopes (IACTs). This next generation observatory will have unprecedented sensitivity in the multi-TeV energy scale, large FOV $\left(45^{\circ}\right)$, daily exposure of the GC, and sufficient angular resolution $\left(<0.5^{\circ}\right)$. The large FOV allows for the continuous observation of several dwarf galaxies in the southern hemisphere, as well as a large fraction of the Galactic DM halo, which reduces the dependence of the search on the DM radial profile. It would, therefore, be sensitive to several different DM profile models (e.g. cuspy or cored).

We investigate in detail the potential of an experiment such as SWGO to measure a DM signal. Based on a "straw man" detector design of SWGO, we derive sensitivity limits to the annihilation and decay of DM particles in the Galactic halo and in Galactic subhalos targets. We show that, together with other contemporaneous gamma-ray observatories, SWGO observations of the GC halo would allow the detection of DM particles with a thermal relic annihilation cross-section $\left(\sim 3 \times 10^{-26} \mathrm{~cm}^{3}\right.$ $\mathrm{s}^{-1}$ ) for masses up to $100 \mathrm{TeV}$. The results included in Section 2 and shown in this presentation were published in [14]. The details about this analysis can be found in the aforementioned reference. In this proceedings we present just the main results.

\section{Searching for Dark Matter in the Galactic Halo with SWGO}

The sensitivity of SWGO to DM annihilation/decay can be found by comparing the number of observable gamma rays with the expected background. This residual background is essentially composed of protons that are misidentified as gamma rays (see ref. [13] for more details). The statistical tool used to derive limits is a 2D (energy and space) joint-likelihood method, where the comparisons between DM and background fluxes are performed in different energy and spatial intervals (or bins) [14].

\subsection{Sensitivity to Dark Matter Annihilation}

Figure 1 shows the expected 95\% C.L. upper limits on $\langle\sigma v\rangle$ versus $M_{\mathrm{DM}}$ for DM particles annihilating into $W^{+} W^{-}, b \bar{b}$ and $\tau^{+} \tau^{-}$[2] assuming an Einasto profile for the GC halo. The

${ }^{1}$ WWW . swgo . org 
sensitivities are shown for 10 years of observations with SWGO. They are compared to the current best limits in the $\mathrm{TeV}$ mass range by H.E.S.S. [5] and to the future sensitivity of CTA-South [10], with 254 and 500 hours of observation of the inner $1^{\circ}$ and $5^{\circ}$ of the Galaxy, respectively, and assuming an Einasto profile in both cases. The current most-stringent Fermi-LAT limits using 15 dwarf spheroidal galaxies (dSphs) are also plotted, as well as the projected sensitivities assuming a total 15 years of observations and a projected sample of $60 \mathrm{dSphs}{ }^{2}$ [9].
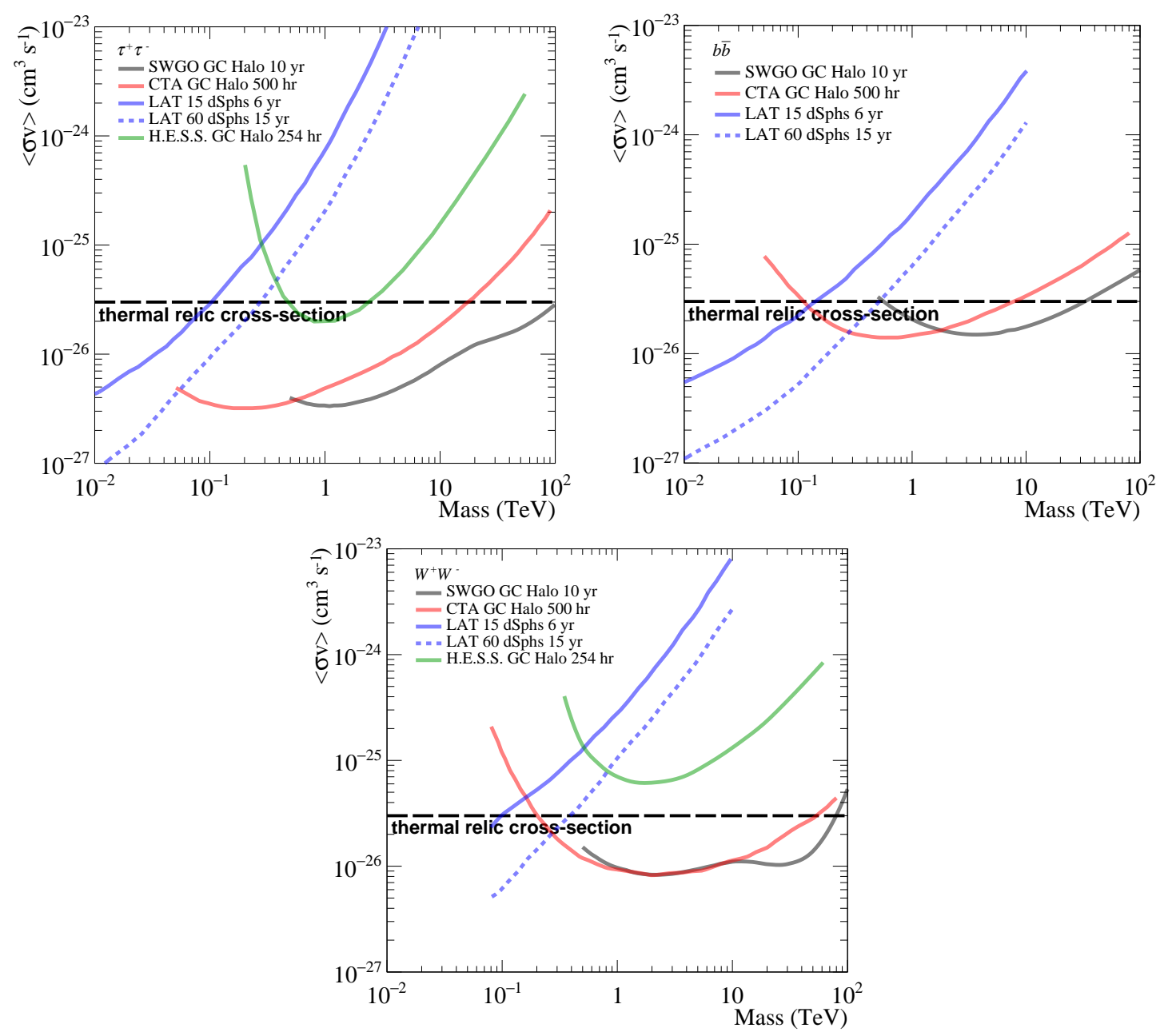

Figure 1: Expected 95\% C.L. upper limit on the velocity-weighted cross section for DM self-annihilation into $\tau^{+} \tau^{-}$(top-left), $b \bar{b}$ (top-right) and $W^{+} W^{-}$(bottom) as a function of $M_{\mathrm{DM}}$, for SWGO and CTA [10] observations of the GC halo. Current Galactic Center H.E.S.S. limits [5] towards the GC halo, and FermiLAT limits towards dwarf galaxies (solid blue line) as well as projected sensitivities (dashed blue line) are also plotted [9]. The nominal value of the thermal-relic cross-section is plotted as well (long-dashed black line). Figure extracted from [14].

A sensitivity to values of $\langle\sigma v\rangle$ smaller than the nominal thermal-relic cross-section is reachable for SWGO in the mass range of $\sim 500 \mathrm{GeV}$ to $\sim 80 \mathrm{TeV}$ for the $W^{+} W^{-}$and $\tau^{+} \tau^{-}$channels, and in

${ }^{2}$ Here we assumed that the Fermi-LAT $W^{+} W^{-}$projected sensitivity scales similar to the $b \bar{b}$ sensitivity. 
the range of $\sim 700 \mathrm{GeV}$ to $\sim 20 \mathrm{TeV}$ for the $b \bar{b}$ channel. SWGO will improve the sensitivity to DM annihilation in the $\mathrm{TeV}$ mass range by more than an order of magnitude with respect to the current observatories, such as H.E.S.S. [5]. It will also be more sensitive than CTA for all DM particles masses above $700 \mathrm{GeV}$ in the $\tau^{+} \tau^{-}$channel, and above $\sim 2.5 \mathrm{TeV}$ in the $b \bar{b}$ channel. In the $W^{+} W^{-}$, SWGO will have a similar sensitivity to CTA in the mass range of $\sim 500 \mathrm{GeV}$ to $\sim 20 \mathrm{TeV}$, and better above $10 \mathrm{TeV}$. Most importantly, the combined sensitivity of SWGO with Fermi-LAT and CTA will be able to probe a thermal-relic cross-section for all WIMP masses between a few $\mathrm{GeV}$ and $\sim 80$ $\mathrm{TeV}$ in most annihilation channels $(\lesssim 20 \mathrm{TeV}$ for $b \bar{b})$.

The fact that CTA and SWGO are both sensitive to DM particles in the TeV mass range with a thermal-relic cross-section means that they could potentially detect DM simultaneously. In the case of a detection, the combination of deep observations of the GC region by SWGO and CTA, would increase confidence in the characterization of the signal if it were found in both [14].

\subsection{Sensitivity to Dark Matter Decay}

Figure 2 shows the expected 95\% C.L. upper limits on the decay lifetime $\tau$ versus $M_{\mathrm{DM}}$ for DM particles decaying into $b \bar{b}$ and $\tau^{+} \tau^{-}$assuming both an Einasto and a Burkert profile for the GC halo. Sensitivities are shown for 10 years of observations with SWGO. CTA sensitivity curves are also shown for 200 hours of observation of an Einasto profile, and decays into $b \bar{b}$ and $\tau^{+} \tau^{-}$[15]. The current most-stringent Fermi-LAT limits based on the observation and modeling of the isotropic gamma-ray background (IGRB) [16] are also plotted. Current limits to DM decay in the GC halo have been provided by HAWC [4], and these are added to Fig. 2 for comparison.
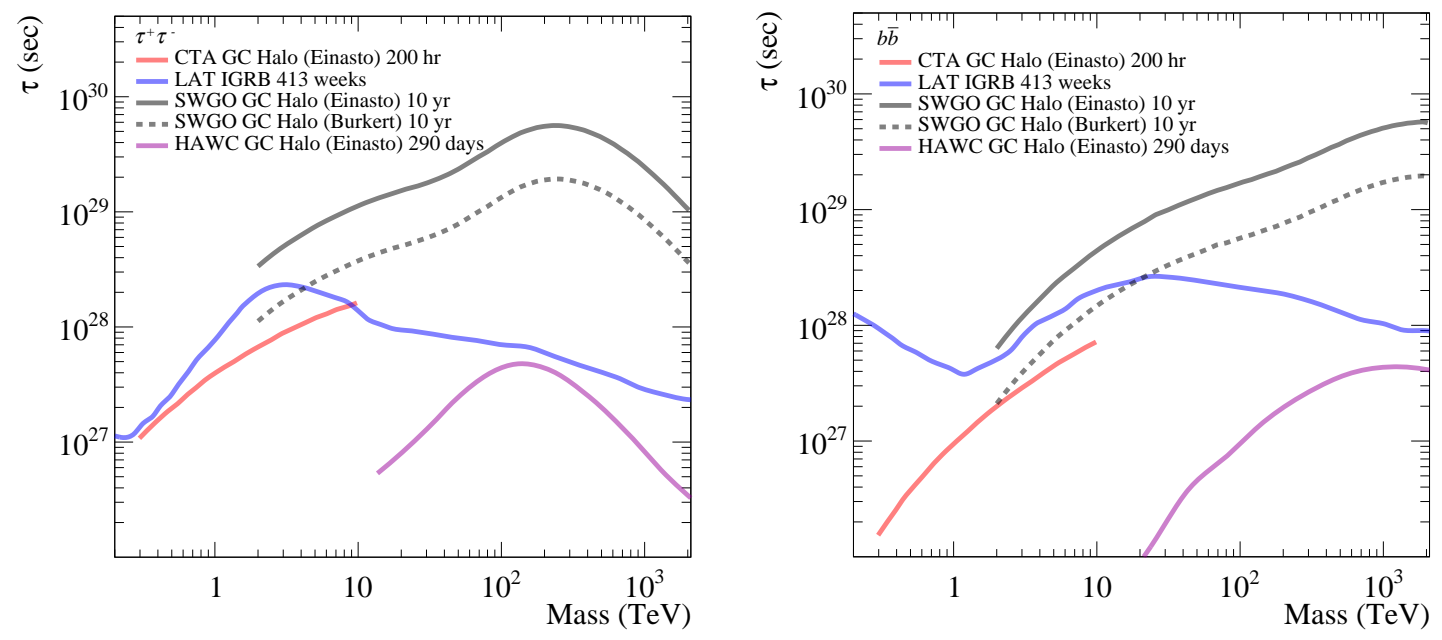

Figure 2: Expected 95\% C.L. lower-limits on the DM decay lifetime into $\tau^{+} \tau^{-}$(left) and $b \bar{b}$ (right) as a function of $M_{\mathrm{DM}}$, for SWGO observations of the GC halo, assuming both an Einasto (solid gray line) and a Burkert (dahsed gray line) profile. CTA sensitivity [15] and HAWC lower-limits [4] are also plotted for observations of an Einasto profile of the GC halo. Fermi-LAT [16] lower-limits from observations of the isotropic gamma-ray background are shown as well. Figure extracted from [14].

SWGO will reach an unprecedented sensitivity in the $\mathrm{TeV}$ mass range, being more sensitive than CTA and Fermi-LAT for all DM particle masses above $\sim 600 \mathrm{GeV}$. With respect to the current limits 

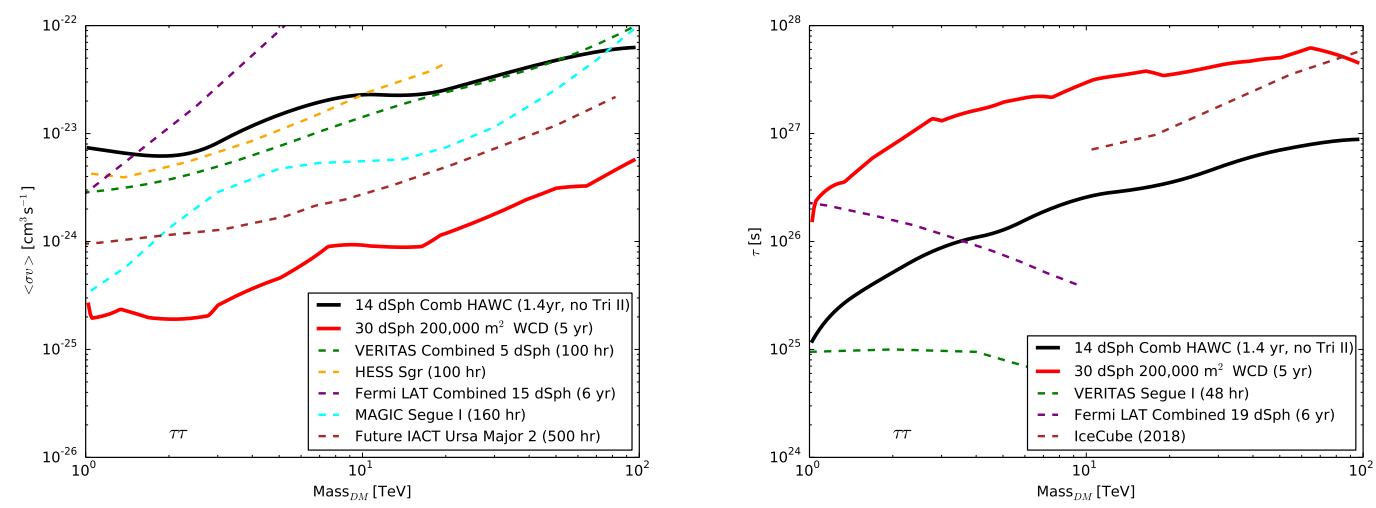

Figure 3: (Left) Expected 95\% confidence level dark-matter-annihilation cross-section upper limits in dSphs with a 200,000 $\mathrm{m}^{2}$ water Cherenkov detector (WCD) (10 times the HAWC area, similar to SWGO). Also shown are the observed dSph limits from VERITAS [8], H.E.S.S. [6], Fermi-LAT [18], MAGIC [7], and the expected limits from CTA [19]. (Right) Expected 95\% confidence level dark-matter decay lifetime lower limits for dark-matter decay in dSphs. Also shown are the observed dSph limits from VERITAS [8], Fermi-LAT [20], and IceCube [21].

from HAWC [4], an improvement of more than two orders of magnitude is expected. A sensitivity to decaying lifetimes larger than $10^{27}$ seconds will be attained for all channels and masses above 1 $\mathrm{TeV}$.

\section{Searching Dark Matter in Galactic Substructure with SWGO}

Dwarf spheroidal galaxies (dSphs) are some of the most dark-matter-dominated objects known. Dozens are known to exist nearby in the Milky Way dark-matter halo. Given their proximity and low astrophysical backgrounds, Milky Way dSphs are excellent targets for searching for gamma-ray emission from dark-matter annihilation or decay. For example, HAWC has derived competitive limits on dark-matter annihilation and decay using $14 \mathrm{dSphs}$ with known dark-matter content [3].

Recent deep observations with wide-field optical imaging surveys have discovered 33 new ultra-faint Milky Way satellites ([17] and references therein), mostly in the Southern Hemisphere. These objects are potentially dark-matter-dominated dSphs, but this needs to be confirmed with spectroscopic follow-up observations. Fifteen of the new satellites have already been spectroscopically confirmed as dSphs. These add to the existing 18 well-characterized dSphs known before these surveys [18].

Figure 3 shows the expected improvement in the dark-matter annihilation and decay limits relative to the current HAWC limits for a HAWC-like array with $10 \times$ better sensitivity in the Southern Hemisphere and an increased number of dSphs. We assume the dark-matter amount and distribution in the new $\mathrm{dSphs}$ matches that of the previously known dSphs. With the next generation of observations, we expect to be able to improve the present limits by nearly an order of magnitude. We also expect such an array's dSph searches to be more sensitive than dSph searches from current and future IACTs like H.E.S.S. and CTA. 
Additionally, with a survey instrument examining the sky every day, additional dSphs that haven't been discovered yet will have already been observed with the instrument's full sensitivity. The Rubin Observatory [22] will survey the Southern Hemisphere sky with unprecedented sensitivity and is expected to find hundreds of new dSphs. Legacy data from SWGO at these locations could easily and immediately be analysed when new dSphs are found.

Two other promising Southern Hemisphere dark-matter targets are the Large (LMC) and Small (SMC) Magellanic Clouds. The LMC is the largest Milky Way satellite and rich in dark matter. It also is likely on its first in-fall, meaning it has not been tidally stripped by the galaxy and still contains most of the its initial dark-matter density. The SMC is in a complicated orbit around the LMC, but also likely on its first in-fall and therefore not tidally stripped. Though its dynamics are more complicated than the LMC, the SMC's rotations curves show that it is dark-matter dominated. Since it is extended by $\sim 10^{\circ}$, a wide-field-of-view observatory would uniquely observe the entire LMC dark-matter subhalo at $\mathrm{TeV}$ energies.

\section{Conclusion}

The Southern Hemisphere is home to many key dark matter targets to search for gamma-ray signals from DM annihilation or decay, such as the inner Galactic halo and several dwarf galaxies. A survey-style instrument with a wide FOV in the Southern Hemisphere, such as SWGO, will be an important tool in searching for such emissions from multi-TeV DM mass. Here we propose a design of SWGO that would be sensitive enough to probe thermal DM for a large range of multi$\mathrm{TeV}$ DM masses and interaction channels when observing the Galactic halo. In addition, we show that such an observatory would produce competitive, if not the best, limits for dark matter from Galactic substructure targets with masses from $100 \mathrm{GeV}$ to several hundreds of TeV. With all of these advantages available, a Southern wide FOV gamma-ray observatory promises to shed new light on the still unknown nature of DM. It will be a critical tool towards a better understanding of this diverse topic in the coming decade.

\section{Acknowledgments}

The SWGO Collaboration acknowledges the support from the agencies and organizations listed here: https://wWW. swgo.org/SWGOWiki/doku.php?id=acknowledgements.

\section{References}

[1] G. Bertone and D. Hooper, Rev. Mod. Phys. 90, no.4, 045002 (2018) doi:10.1103/RevModPhys.90.045002

[2] M. Cirelli, G. Corcella, A. Hektor, G. Hutsi, M. Kadastik, P. Panci, M. Raidal, F. Sala and A. Strumia, JCAP 03, 051 (2011) [erratum: JCAP 10, E01 (2012)]

[3] A. Albert et al. [HAWC], Astrophys. J. 853, no.2, 154 (2018) doi:10.3847/1538-4357/aaa6d8

[4] A. U. Abeysekara et al. [HAWC], JCAP 02, 049 (2018) doi:10.1088/1475-7516/2018/02/049 
[5] H. Abdallah et al. [H.E.S.S.], Phys. Rev. Lett. 117, no.11, 111301 (2016) doi:10.1103/PhysRevLett.117.111301

[6] A. Abramowski et al. [H.E.S.S.], Phys. Rev. D 90, 112012 (2014) doi:10.1103/PhysRevD.90.112012

[7] M. L. Ahnen et al. [MAGIC and Fermi-LAT], JCAP 02, 039 (2016) doi:10.1088/1475$7516 / 2016 / 02 / 039$

[8] S. Archambault et al. [VERITAS], Phys. Rev. D 95, no.8, 082001 (2017) doi:10.1103/PhysRevD.95.082001

[9] A. Albert et al. [Fermi-LAT and DES], Astrophys. J. 834, no.2, 110 (2017) doi:10.3847/1538$4357 / 834 / 2 / 110$

[10] B. S. Acharya et al. [CTA Consortium], doi:10.1142/10986 [arXiv:1709.07997 [astro-ph.IM]].

[11] D. Z. He, X. J. Bi, S. J. Lin, P. F. Yin and X. Zhang, Phys. Rev. D 100, no.8, 083003 (2019) doi:10.1103/PhysRevD.100.083003

[12] X. Bai, B. Y. Bi, X. J. Bi, Z. Cao, S. Z. Chen, Y. Chen, A. Chiavassa, X. H. Cui, Z. G. Dai and D. della Volpe, et al. [arXiv:1905.02773 [astro-ph.HE]].

[13] H. Schoorlemmer [SWGO], PoS ICRC2019, 785 (2020) doi:10.22323/1.358.0785

[14] A. Viana, H. Schoorlemmer, A. Albert, V. de Souza, J. P. Harding and J. Hinton, JCAP 12, 061 (2019) doi:10.1088/1475-7516/2019/12/061

[15] M. Pierre, J. M. Siegal-Gaskins and P. Scott, JCAP 06, 024 (2014) [erratum: JCAP 10, E01 (2014)] doi:10.1088/1475-7516/2014/10/E01

[16] T. Cohen, K. Murase, N. L. Rodd, B. R. Safdi and Y. Soreq, Phys. Rev. Lett. 119, no.2, 021102 (2017) doi:10.1103/PhysRevLett.119.021102

[17] L. E. Strigari, Rept. Prog. Phys. 81, no.5, 056901 (2018) doi:10.1088/1361-6633/aaae16

[18] M. Ackermann et al. [Fermi-LAT], Phys. Rev. Lett. 115, no.23, 231301 (2015) doi:10.1103/PhysRevLett.115.231301

[19] V. Lefranc, G. A. Mamon and P. Panci, JCAP 09, 021 (2016) doi:10.1088/1475$7516 / 2016 / 09 / 021$

[20] M. G. Baring, T. Ghosh, F. S. Queiroz and K. Sinha, Phys. Rev. D 93, no.10, 103009 (2016) doi:10.1103/PhysRevD.93.103009

[21] M. G. Aartsen et al. [IceCube], Eur. Phys. J. C 78, no.10, 831 (2018) doi:10.1140/epjc/s10052018-6273-3

[22] D. Alonso et al. [LSST Dark Energy Science], [arXiv:1809.01669 [astro-ph.CO]]. 


\section{Full Authors List: SWGO Collaboration}

P. Abreu ${ }^{1}$, A. Albert ${ }^{2}$, E. O. Angüner ${ }^{3}$, C. Arcaro $^{4}$, L.H. Arnaldi ${ }^{5}$, J.C. Arteaga-Velázquez ${ }^{6}$, P. Assis ${ }^{1}$, A. Bakalová ${ }^{7}$, U. Barres de Almeida ${ }^{8}$, I. Batković ${ }^{4}$, J. Bellido ${ }^{9}$, E. Belmont-Moreno ${ }^{10}$, F. Bisconti ${ }^{11}$, A. Blanco ${ }^{1}$, M. Bohacova ${ }^{7}$, E. Bottacini ${ }^{4}$, T. Bretz ${ }^{12}$, C. Brisbois ${ }^{13}$, P. Brogueira ${ }^{1}$, A.M. Brown ${ }^{14}$, T. Bulik ${ }^{15}$, K. S. Caballero Mora ${ }^{16}$, S. M. Campos ${ }^{17}$ A. Chiavassa ${ }^{11}$, L. Chytka ${ }^{7}$, R. Conceição ${ }^{1}$, G. Consolati ${ }^{18}$, J. Cotzomi Paleta ${ }^{19}$, S. Dasso ${ }^{20}$, A. De Angelis ${ }^{4}$, C. R. De Bom ${ }^{8}$, E. de la Fuente ${ }^{21}$, V. de Souza ${ }^{22}$, D. Depaoli ${ }^{11}$, G. Di Sciascio ${ }^{23}$, C. O. Dib ${ }^{24}$, D. Dorner ${ }^{25}$, M. Doro ${ }^{4}$, M. Du Vernois ${ }^{26}$, T. Erginn $^{27}$, K. L. Fan ${ }^{13}$, N. Fraija ${ }^{8}$, S. Funk $^{28}$, J. I. García ${ }^{17}$, J. A. García-González ${ }^{29}$, S. T. García Roca ${ }^{9}$, G. Giacinti ${ }^{30}$, H. Goksu ${ }^{30}$, B. S. González ${ }^{1}$, F. Guarino ${ }^{31}$, A. Guillén ${ }^{32}$,

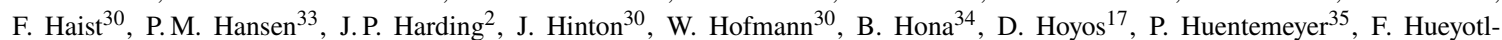
Zahuantitla $^{16}$ A. Insolia ${ }^{36}$, P. Janecek ${ }^{7}$, V. Joshi ${ }^{28}$, B. Khelifi ${ }^{37}$, S. Kunwar ${ }^{30}$, G. La Mura ${ }^{1}$, J. Lapington ${ }^{38}$, M. R. Laspiur ${ }^{17}$, F. Leit1 ${ }^{28}$, F. Longo ${ }^{39}$, L. Lopes ${ }^{1}$, R. Lopez-Coto ${ }^{4}$, D. Mandat ${ }^{7}$, A. G. Mariazzi ${ }^{33}$, M. Mariotti ${ }^{4}$, A. Marques Moraes ${ }^{8}$, J. MartínezCastro $^{40}$, H. Martínez-Huerta ${ }^{41}$, S. May ${ }^{42}$, D. G. Melo ${ }^{43}$, L. F. Mendes ${ }^{1}$, L. M. Mendes ${ }^{1}$, T. Mineeva ${ }^{24}$, A. Mitchell ${ }^{44}$, S. Mohan $^{35}$, O. G. Morales Olivares ${ }^{16}$, E. Moreno-Barbosa ${ }^{19}$, L. Nellen ${ }^{45}$, V. Novotny ${ }^{7}$, L. Olivera-Nieto ${ }^{30}$, E. Orlando ${ }^{39}$, M. Pech $^{7}$, A. Pichel $^{20}$, M. Pimenta ${ }^{1}$, M. Portes de Albuquerque ${ }^{8}$, E. Prandini ${ }^{4}$, M. S. Rado Cuchills ${ }^{9}$, A. Reisenegger ${ }^{46}$, B. Reville ${ }^{30}$, C. D. Rho ${ }^{47}$, A. C. Rovero ${ }^{20}$ E. Ruiz-Velasco ${ }^{30}$, G. A. Salazar ${ }^{17}$, A. Sandoval ${ }^{10}$, M. Santander ${ }^{42}$, H. Schoorlemmer ${ }^{30}$, F. Schüssler ${ }^{48}$, V. H. Serrano ${ }^{17}$, R. C. Shellard ${ }^{8}$, A. Sinha ${ }^{49}$, A. J. Smith ${ }^{13}$, P. Surajbali ${ }^{30}$, B. Tomé ${ }^{1}$, I. Torres Aguilar ${ }^{50}$, C. van Eldik ${ }^{28}$, I. D. Vergara-Quispe ${ }^{33}$, A. Viana ${ }^{22}$, J. Vícha ${ }^{7}$, C. F. Vigorito ${ }^{11}$, X. Wang ${ }^{35}$, F. Werner ${ }^{30}$, R. White ${ }^{30}$, M. A. Zamalloa Jara ${ }^{9}$

${ }^{1}$ Laboratório de Instrumentação e Física Experimental de Partículas (LIP), Av. Prof. Gama Pinto 2, 1649-003 Lisboa, Portugal

${ }^{2}$ Physics Division, Los Alamos National Laboratory, P.O. Box 1663, Los Alamos, NM 87545, United States

${ }^{3}$ Aix Marseille Univ, CNRS/IN2P3, CPPM, 163 avenue de Luminy - Case 902, 13288 Marseille cedex 09, France

${ }^{4}$ University of Padova, Department of Physics and Astronomy \& INFN Padova, Via Marzolo 8 - 35131 Padova, Italy

${ }^{5}$ Centro Atómico Bariloche, Comisión Nacional de Energía Atómica, S. C. de Bariloche (8400), RN, Argentina

${ }^{6}$ Universidad Michoacana de San Nicolás de Hidalgo, Calle de Santiago Tapia 403, Centro, 58000 Morelia, Mich., México

${ }^{7}$ FZU, Institute of Physics of the Czech Academy of Sciences, Na Slovance 1999/2, 18200 Praha 8, Czech Republic

${ }^{8}$ Centro Brasileiro de Pesquisas Físicas, R. Dr. Xavier Sigaud, 150 - Rio de Janeiro - RJ, 22290-180, Brazil

${ }^{9}$ Academic Department of Physics - Faculty of Sciences - Universidad Nacional de San Antonio Abad del Cusco (UNSAAC), Av. de la Cultura, 733, Pabellón C-358, Cusco, Peru

${ }^{10}$ Instituto de Física, Universidad Nacional Autónoma de México, Sendero Bicipuma, C.U., Coyoacán, 04510 Ciudad de México, CDMX, México

${ }^{11}$ Dipartimento di Fisica, Università degli Studi di Torino, Via Pietro Giuria 1, 10125, Torino, Italy

${ }^{12}$ RWTH Aachen University, Physics Institute 3, Otto-Blumenthal-Straße, 52074 Aachen, Germany

${ }^{13}$ University of Maryland, College Park, MD 20742, United States

${ }^{14}$ Durham University, Stockton Road, Durham, DH1 3LE, United Kingdom

${ }^{15}$ Astronomical Observatory, University of Warsaw, Aleje Ujazdowskie 4, 00478 Warsaw, Poland

${ }^{16}$ Facultad de Ciencias en Física y Matemáticas UNACH, Boulevard Belisario Domínguez, Km. 1081, Sin Número, Terán, Tuxtla Gutiérrez, Chiapas, México

${ }^{17}$ Facultad de Ciencias Exactas, Universidad Nacional de Salta, Avda. Bolivia No 5150, (4400) Salta Capital, Argentina

${ }^{18}$ Department of Aerospace Science and Technology, Politecnico di Milano, Via Privata Giuseppe La Masa, 34, 20156 Milano MI, Italy

${ }^{19}$ Facultad de Ciencias Físico Matemáticas, Benemérita Universidad Autónoma de Puebla, C.P. 72592, México

${ }^{20}$ Instituto de Astronomia y Fisica del Espacio (IAFE, CONICET-UBA), Casilla de Correo 67 - Suc. 28 (C1428ZAA), Ciudad

Autónoma de Buenos Aires, Argentina

${ }^{21}$ Universidad de Guadalajara, Blvd. Gral. Marcelino García Barragán 1421, Olímpica, 44430 Guadalajara, Jal., México

22 Instituto de Física de São Carlos, Universidade de São Paulo, Avenida Trabalhador São-carlense, no 400, Parque Arnold Schimidt CEP 13566-590, São Carlos - São Paulo - Brazil

${ }^{23}$ INFN - Roma Tor Vergata and INAF-IAPS, Via del Fosso del Cavaliere, 100, 00133 Roma RM, Italy

${ }^{24}$ Dept. of Physics and CCTVal, Universidad Tecnica Federico Santa Maria, Avenida España 1680, Valparaíso, Chile

${ }^{25}$ Universität Würzburg, Institut für Theoretische Physik und Astrophysik, Emil-Fischer-Str. 31, 97074 Würzburg, Germany

${ }^{26}$ Department of Physics, and the Wisconsin IceCube Particle Astrophysics Center (WIPAC), University of Wisconsin, 222 West Washington Ave., Suite 500, Madison, WI 53703, United States

27 TUBITAK Space Technologies Research Institute, ODTU Campus, 06800, Ankara, Turkey

${ }^{28}$ Friedrich-Alexander-Universität Erlangen-Nürnberg, Erlangen Centre for Astroparticle Physics, Erwin-Rommel-Str. 1, D 91058 Erlangen, Germany

${ }^{29}$ Tecnologico de Monterrey, Escuela de Ingeniería y Ciencias, Ave. Eugenio Garza Sada 2501, Monterrey, N.L., 64849, México

${ }^{30}$ Max-Planck-Institut für Kernphysik, P.O. Box 103980, D 69029 Heidelberg, Germany

${ }^{31}$ Università di Napoli "Federico II", Dipartimento di Fisica "Ettore Pancini", and INFN Napoli, Complesso Universitario di Monte Sant’Angelo - Via Cinthia, 21 - 80126 - Napoli, Italy

${ }^{32}$ University of Granada, Campus Universitario de Cartuja, Calle Prof. Vicente Callao, 3, 18011 Granada, Spain 
${ }^{33}$ IFLP, Universidad Nacional de La Plata and CONICET, Diagonal 113, Casco Urbano, B1900 La Plata, Provincia de Buenos Aires, Argentina

34 University of Utah, 201 Presidents' Cir, Salt Lake City, UT 84112, United States

${ }^{35}$ Michigan Technological University, 1400 Townsend Drive, Houghton, MI 49931, United States

${ }^{36}$ Dipartimento di Fisica e Astronomia "E. Majorana", Catania University and INFN, Catania, Italy

${ }^{37}$ APC-IN2P3/CNRS, Université de Paris, Bâtiment Condorcet, 10 rue A.Domon et Léonie Duquet, 75205 PARIS CEDEX 13, France

${ }^{38}$ University of Leicester, University Road, Leicester LE1 7RH, United Kingdom

${ }^{39}$ Department of Physics, University of Trieste and INFN Trieste, via Valerio 2, I-34127, Trieste, Italy

${ }^{40}$ Centro de Investigación en Computación, Instituto Politécnico Nacional, Av. Juan de Dios Bátiz S/N, Nueva Industrial Vallejo, Gustavo A. Madero, 07738 Ciudad de México, CDMX, México

${ }^{41}$ Department of Physics and Mathematics, Universidad de Monterrey, Av. Morones Prieto 4500, San Pedro Garza García 66238, N.L., México

42 Department of Physics and Astronomy, University of Alabama, Gallalee Hall, Tuscaloosa, AL 35401, United States

${ }^{43}$ Instituto de Tecnologías en Detección y Astropartículas (CNEA-CONICET-UNSAM), Av. Gral Paz 1499 - San Martín - Pcia. de Buenos Aires, Argentina

${ }^{44}$ Department of Physics, ETH Zurich, CH-8093 Zurich, Switzerland

45 Instituto de Ciencias Nucleares, Universidad Nacional Autónoma de México (ICN-UNAM), Cto. Exterior S/N, C.U., Coyoacán, 04510 Ciudad de México, CDMX, México

${ }^{46}$ Departamento de Física, Facultad de Ciencias Básicas, Universidad Metropolitana de Ciencias de la Educación, Av. José Pedro Alessandri 774, Ñuñoa, Santiago, Chile

${ }^{47}$ Department of Physics, University of Seoul, 163 Seoulsiripdaero, Dongdaemun-gu, Seoul 02504, Republic of Korea

${ }^{48}$ Institut de recherche sur les lois fondamentales de l'Univers (IRFU), CEA, Université Paris-Saclay, F-91191 Gif-sur-Yvette, France

${ }^{49}$ Laboratoire Univers et Particules de Montpellier, CNRS, Université de Montpelleir, F-34090 Montpellier, France

${ }^{50}$ Instituto Nacional de Astrofísica, Óptica y Electrónica (INAOE), Luis Enrique Erro 1, Puebla, México 